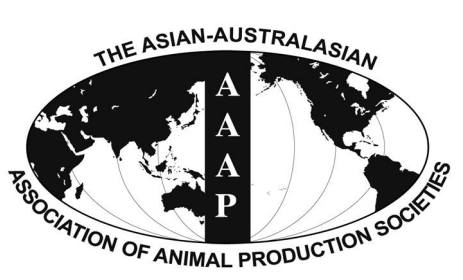

Open Access

Asian Australas. J. Anim. Sci.

Vol. 28, No. 11 : 1565-1572 November 2015

http://dx.doi.org/10.5713/ajas.15.0462

www.ajas.info

pISSN 1011-2367 elSSN 1976-5517

\title{
Embryo Aggregation Promotes Derivation Efficiency of Outgrowths from Porcine Blastocysts
}

\author{
Sang-Goo Lee ${ }^{1,2, a}$, Jin-Kyu Park ${ }^{1,3, a}$, Kwang-Hwan Choi ${ }^{1}$, Hye-Young Son ${ }^{1}$, and Chang-Kyu Lee ${ }^{1,4, *}$ \\ ${ }^{1}$ Department of Agricultural Biotechnology, Animal Biotechnology Major, and \\ Research Institute for Agriculture and Life Science, Seoul National University, Seoul 151-921, Korea
}

\begin{abstract}
Porcine embryonic stem cells (pESCs) have become an advantageous experimental tool for developing therapeutic applications and producing transgenic animals. However, despite numerous reports of putative pESC lines, deriving validated pESC lines from embryos produced in vitro remains difficult. Here, we report that embryo aggregation was useful for deriving pESCs from in vitro-produced embryos. Blastocysts derived from embryo aggregation formed a larger number of colonies and maintained cell culture stability. Our derived cell lines demonstrated expression of pluripotent markers (alkaline phosphatase, Oct4, Sox2, and Nanog), an ability to form embryoid bodies, and the capacity to differentiate into the three germ layers. A cytogenetic analysis of these cells revealed that all lines derived from aggregated blastocysts had normal female and male karyotypes. These results demonstrate that embryo aggregation could be a useful technique to improve the efficiency of deriving ESCs from in vitro-fertilized pig embryos, studying early development, and deriving pluripotent ESCs in vitro in other mammals. (Key Words: Embryo Aggregation, Embryonic Stem Cells, In vitro-produced Embryos, Embryo Quality, Derivation Efficiency, Pig)
\end{abstract}

\section{INTRODUCTION}

Establishing embryonic stem cells (ESCs) in domestic animals aids in the understanding of embryonic biology and improves the outcomes of regenerative medicine studies using stem cells. The pig is an ideal animal model for biomedical research, as it is immunologically and physiologically similar to humans. The pig has potential for xenografting and cell therapy regenerative medicine studies (Talbot and Blomberg Le, 2008). Thus, numerous efforts

\footnotetext{
* Corresponding Author: Chang-Kyu Lee. Tel: +82-2-880-4805, Fax:+82-2-873-4805, E-mail: leeck@snu.ac.kr

${ }^{2}$ Division of Genetics, Department of Medicine, Brigham and Women's Hospital, Harvard Medical School, Boston, MA 02115 , USA.

${ }^{3}$ Division of Animal Sciences and Bond Life Sciences Center, University of Missouri, Columbia, MO 65211, USA.

${ }^{4}$ Institute of Green Bio Science and Technology, Seoul National University, Pyeong Chang 232-916, Korea.

${ }^{a}$ Both authors contributed equally to this work.

Submitted May 26, 2015; Revised Jul. 4, 2015; Accepted Jul. 17, 2015
}

have been made to establish pluripotent stem cells from porcine embryos. Although authentic porcine embryonic stem cell (pESC) lines have not been derived, a number of putative $\mathrm{pESCs}$ have been established from various porcine embryos, including in vitro-produced and in vivo-derived embryos and parthenogenetic embryos, as well as in vitroaggregated and somatic cell nuclear transfer (SCNT) embryos (Li et al., 2004; Kim et al., 2010; Vassiliev et al., 2010; Park et al., 2013). However, most of these showed limited pluripotency compared with that of mouse embryonic stem cells (mESCs). In particular, these putative pESCs do not form teratomas or display germ-line chimerism.

We previously conducted a comparative study of stem cells reprogrammed in pigs using epiblast stem cell (EpiSC)-like pESC lines derived from porcine embryos of various origins, including in vitro fertilized (IVF), in vivoderived, IVF-aggregated, and parthenogenetic embryos (Park et al., 2013). These previous results showed that stem cell lines derived from porcine pre-implantation embryos from various origins exhibited flattened morphology and 
had the ability to differentiate in vitro and to activate the fibroblast growth factor and nodal/activin pathways. They belong to the primed state, rather than the naïve state, cell category. Alberio et al. reported a porcine EpiSC line from embryonic day 10.5 to 12 porcine embryos that activated nodal/activin signaling to maintain their pluripotency (Alberio et al., 2010). Thus, putative pESCs are similar to porcine EpiSCs but different from mESCs with respect to morphology and the pathways activated.

In humans as well as in pigs, in vitro culture conditions are suboptimal and result in low blastocyst rates or blastocysts with low cell numbers and a lack of a distinct inner cell mass (ICM) (Pickering et al., 2003). IVF embryos show less developmental competence and poorer ICM quality than do in vivo-produced embryos (Bavister, 2004). Embryo quality also affects the efficiency of ESC derivation because ESCs are derived from the ICM (Keefer et al., 2007). An embryo aggregation technique has been used to improve the quality of IVF embryos. Early embryo aggregation studies produced transgenic chimeras in mice (Nagy et al., 1993; Wood et al., 1993) and cattle (Boediono et al., 1993; Boediono et al., 1999). Subsequent studies demonstrated the advantages of aggregation to enhance in vitro development (Tang and West, 2000) as a means of rescuing cell blocks (Neganova et al., 2000) and overcoming early developmental obstacles in cloning by SCNT (Boiani et al., 2003). Our previous study demonstrated that aggregating porcine IVF embryos increased the in vitro development rate and total cell numbers, with a proportional increase in both ICM and trophectoderm (TE) cells and expression of mRNAs related to embryo quality (Lee et al., 2007).

Therefore, the present study evaluated whether these blastocysts could be used to derive pESCs and assessed the effects of deriving pES-like cells from IVF-aggregated blastocysts. The results showed that our cell lines expressed pluripotent markers, had normal karyotypes, and retained the potential to differentiate into the three germ layers under in vitro conditions. We demonstrated that embryo aggregation is an efficient technique to overcome the obstacles produced by in vitro methods and that the techniques can be used to study early development and derive authentic pluripotent pESCs.

\section{MATERIALS AND METHODS}

\section{Animal welfare}

The authors assert that all procedures contributing to this work comply with the ethical standards of the relevant national and institutional guides on the care and use of laboratory animals. The care and experimental use of pigs and mice was approved by the Institute of Laboratory Animal Resources, Seoul National University (SNU-
140217-3).

\section{Embryo aggregation and in vitro culture}

Oocytes and IVF embryos were produced using the method described in our previous study (Lee et al., 2007). After IVF for 2 days, the zona pellucida of four-cell-stage embryos was removed with acidic Tyrode's solution (Sigma-Aldrich, St. Louis, MO, USA; http://www. sigmaaldrich.com). Clusters of depressions were generated in the bottom of a culture dish using gentle pressure with a darning needle (BLS, Budapest, Hungary http://www.blsltd.com) and were covered with microdrops $(40 \mu \mathrm{L})$ of North Carolina State University-23 medium supplemented with $4 \mathrm{mg} / \mathrm{mL}$ fatty acid-free bovine serum albumin (Sigma-Aldrich, USA) (Petters and Wells, 1993), overlaid with oil, and incubated for at least $1 \mathrm{~h}$ prior to use. Zonafree embryos were placed in groups of two or three into each microwell for aggregation. Non-aggregated $(1 \times)$, twoembryo aggregated $(2 \times)$, and three-embryo aggregated $(3 \times)$ four-cell-stage embryos were cultured in parallel in separate drops within the same dish. The aggregates were cultured at $39^{\circ} \mathrm{C}$ in a $5 \% \mathrm{CO}_{2}, 5 \% \mathrm{O}_{2}$, and $90 \% \mathrm{~N}_{2}$ atmosphere for 2 days and then added to microwells containing 10\% fetal bovine serum (FBS; collected and processed in Canada; Hyclone, Logan, UT, USA; www.hyclone.com) (Kim et al., 2004).

\section{Derivation of outgrowths from porcine blastocysts}

Porcine blastocysts were cultured on mitotically inactivated mouse embryonic fibroblasts (MEFs) in pESC medium, which was a 50:50 mixture of low-glucose Dulbecco's modified Eagle's medium (Gibco/Invitrogen, Carlsbad, CA, USA; www.invitrogen.com) and Ham's F10 medium (Gibco), supplemented with 15\% FBS, $2 \mathrm{mM}$ Glutamax (Gibco), $0.1 \mathrm{mM} \beta$-mercaptoethanol (Gibco), 1× Minimum Essential Medium with nonessential amino acids (Gibco), 1× antibiotic/antimycotic (Gibco) containing cytokines, $40 \mathrm{ng} / \mathrm{mL}$ human recombinant stem cell factor (R\&D Systems, Minneapolis, MN, USA; www. rndsystems.com), and $20 \mathrm{ng} / \mathrm{mL}$ human recombinant basic fibroblast growth factor (R\&D Systems, USA). Two seeding methods were used to establish the pluripotent cell lines: intact blastocyst-stage embryos either were cultured directly on MEFs or were subjected to mechanical dissection under a microscope using pulled glass pipettes to separate the ICM from the TE prior to seeding. ES-like primary colonies derived from day 7 in vivo-produced and day 8 in vitro-hatched blastocysts were observed after 5 to 7 days of culture. These ES-like cell colonies were mechanically dissociated into several clumps using pulled glass pipettes 10 to 15 days after seeding. The dissociated clumps were re-seeded on fresh inactivated MEFs, and the subsequent ES-like cell lines were routinely passaged via 
the pulled glass pipette method every 5 to 7 days. All cells were cultured under humidified conditions at $37^{\circ} \mathrm{C}$ with $5 \%$ $\mathrm{CO}_{2}$.

\section{Alkaline phosphatase activity and immunocytochemistry analyses}

For alkaline phosphatase (AP) staining, the cells were fixed in $4 \%$ paraformaldehyde for $15 \mathrm{~min}$. After washing, the fixed cells were stained with a solution containing a nitro blue tetrazolium chloride and 5-bromo-4-chloro-3indolyl phosphate toluidine salt stock solution (Roche, Madison, WI, USA; www.roche.com) in a buffer solution for $30 \mathrm{~min}$ at room temperature. The fixed cells were washed and permeabilized (for intracellular markers only) with $0.2 \%$ Triton X-100 (Sigma, USA) for $5 \mathrm{~min}$ for the immunocytochemistry (ICC) analysis of undifferentiated or differentiated cells. The washed cells were co-incubated with blocking solution (10\% goat serum in Dulbecco's phosphate-buffered saline) and primary antibodies overnight at $4^{\circ} \mathrm{C}$. The primary antibodies used were Oct4 (SC-9081, Santa Cruz Biotechnology, Santa Cruz, CA, USA; www.scbt.com 1:100), Nanog (SC-33759, Santa Cruz Biotechnology, USA, 1:100), Sox2 (AB5603, Millipore, Temecula, CA, USA; www. millipore.com, 1:200), neurofilament (MAB1615, Millipore, USA, 1:200), vimentin (MAB3400, Millipore, USA, 1:200), and cytokeratin17 (MAB1625, Millipore, USA, 1:200). The cells were washed, incubated with the appropriate secondary antibody, and stained with Hoechst 33342 or propidium iodide. The stained cells were examined under a confocal microscope using ZEN 2012 Light Edition (Carl Zeiss, Zena, Germany; www.zeiss.com).

\section{Embryoid body formation and in vitro differentiation}

The ES-like cell lines were removed from MEFs, mechanically dissociated with glass pipettes, and cultured in pESC medium without cytokines using the hanging drop method to evaluate differentiation potential. ES-like cells forming typical embryoid bodies (EBs) after 5 days were transferred to confocal dishes coated with $0.1 \%$ gelatin and allowed to further differentiate for 2 to 3 weeks.

\section{Reverse transcriptase-polymerase chain reaction analysis}

Total RNA from individual samples was extracted using TRIZOL reagent (Invitrogen, USA) according to the manufacturer's instructions to analyze the gene expression patterns of undifferentiated and differentiated cells. cDNA was synthesized using a High Capacity RNA-to-cDNA Kit (Applied Biosystems, Foster City, CA, USA; www.appliedbiosystems.com) according to the manufacturer's instructions, producing a final volume of 20 $\mu \mathrm{L}$. Polymerase chain reaction (PCR) amplifications were performed using the $2 \times$ PCR Master Mix Solution (i-MAX II, iNtRON Biotechnology, Daejeon, Korea; http://eng.intronbio.com/) in a total reaction volume of 20 $\mu \mathrm{L}$, containing $1 \mu \mathrm{L}$ cDNA, $1 \mu \mathrm{L}$ of each primer,_and $7 \mu \mathrm{L}$ distilled water. The conditions and primers used are listed in Table 1 .

\section{Karyotyping}

Standard G-banding chromosome and cytogenetic analyses were carried out at Samkwang Medical Laboratories (Seoul, Korea; www.smlab.co.kr).

\section{Statistical analysis}

The statistical analysis was performed using the " $R$ " program (http://www.R-project.org). Aggregated embryo development to the blastocyst stage was analyzed using the two-tailed Fisher's exact test. Treatment differences were considered significant when the $\mathrm{p}$-value was $<0.05$.

\section{RESULTS}

\section{Outgrowths derived from porcine blastocysts}

We observed blastocyst surface morphology and blastocysts attaching onto the feeder cell layer under high magnification to identify the early outgrowth process in porcine blastocysts. As shown in Figure 1A and 1B, giant cells were observed on the surface section of the blastocysts, which were the so-called TE. A mass of cells, the so-called ICM, was present inside the blastocysts. The cells in the ICM were smaller than those of the TE. The giant cells shown in Figure 1C initially proliferated 3 days after seeding the blastocysts and then gradually died out or

Table 1. Reverse transcription-polymerase chain reaction conditions and primers

\begin{tabular}{|c|c|c|c|}
\hline Genes & Primer sequences & Annealing temperature $\left({ }^{\circ} \mathrm{C}\right)$ & Products size (bp) \\
\hline \multirow[t]{2}{*}{ Oct4 } & 5'-AACGATCAAGCAGTGACTATTCG-3' & 60 & 153 \\
\hline & 5'-GAGTACAGGGTGGTGAAGTGAGG-3' & & \\
\hline \multirow[t]{2}{*}{ Nanog } & 5'-AATCTTCACCAATGCCTGAG-3' & 60 & 141 \\
\hline & 5'-GGCTGTCCTGAATAAGCAGA-3' & & \\
\hline \multirow[t]{2}{*}{ Sox 2} & 5'-CGGCGGCAGGATCGGC-3' & 60 & 113 \\
\hline & 5'-GAGCTCCGCGAGGAAAA-3' & & \\
\hline \multirow[t]{2}{*}{$\beta$-Actin } & 5'-GTGGACATCAGGAAGGACCTCTA-3' & 60 & 137 \\
\hline & 5'-ATGATCTTGATCTTCATGGTGCT-3' & & \\
\hline
\end{tabular}




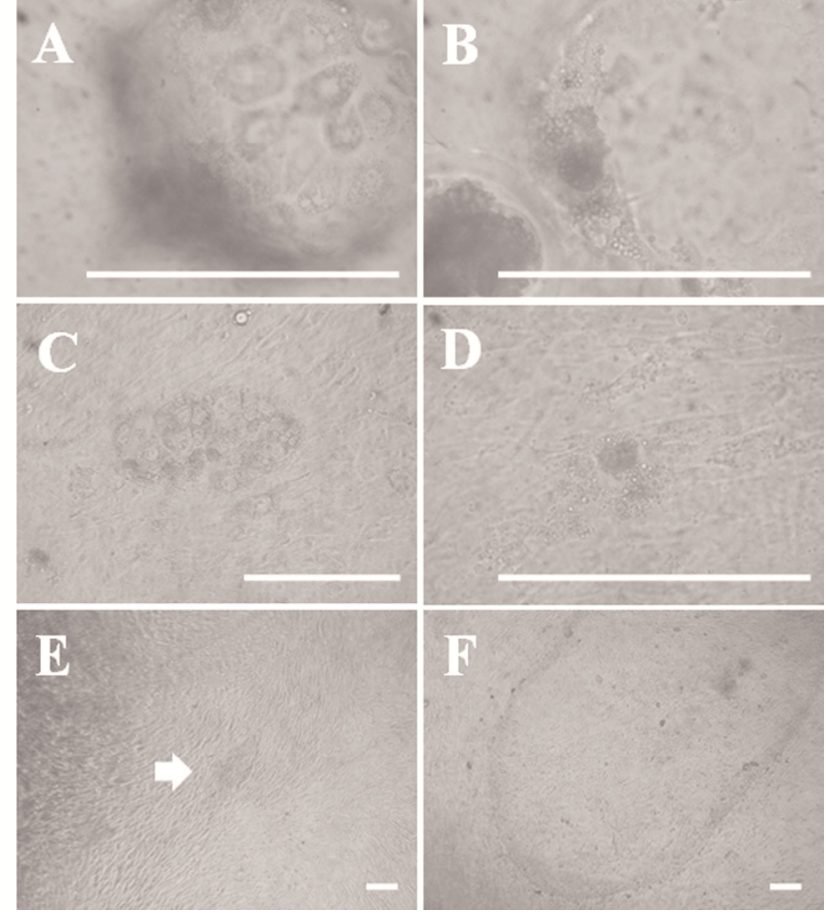

Figure 1. Outgrowths derived from porcine blastocysts. (A) Morphology of trophectoderm (TE)-like giant cells in a blastocyst surface section. (B) Morphology of an inner cell mass (ICM)-like mass of cells inside a blastocyst. (C) Giant cells attached to feeder layer cells 3 days after blastocyst seeding. (D) The mass of cells presumed to be the ICM is attached to the feeder layer. (E) Morphology of an embryonic stem (ES)-like primary colony derived from a porcine embryo. (F) Late morphology of an ESlike primary colony derived from a porcine embryo. Scale bars = $100 \mu \mathrm{m}$.

disappeared within a few days, except the mass of cells presumed to be the ICM (Figure 1C and 1D). We observed small primary colonies with a compact multi-layer morphology after about 7 days (Figure 1E). However, the multi-layer morphology changed to mono-layer morphology after 3 to 4 days. Outgrowths were passaged onto a new feeder layer about 14 days after seeding (Figure $1 \mathrm{~F})$. We passaged the cell lines every 5 days. We have been able to establish ES-like cell lines from various embryos, such as in vivo-derived, IVF, and IVF-aggregated $(3 \times)$ blastocysts, using these methods. The efficiencies of all established cell lines are summarized in Table 2. We confirmed similar primary colony morphology and a typical morphology among these cell lines.

\section{Effect of embryo aggregation on deriving pES-like cells from in vitro fertilized blastocysts}

Non-aggregated $(1 \times)$ and aggregated $(2 \times$ and $3 \times)$ blastocysts were produced in parallel in separate drops under the same conditions while we were determining the effects of embryo aggregation on deriving outgrowths from IVF blastocysts. The total numbers of aggregated embryos
Table 2. Efficiency of the porcine embryonic stem (ES)-like cell lines derived from embryos with different origins

\begin{tabular}{lcc}
\hline Embryo origins & $\begin{array}{c}\text { No. of } \\
\text { blastocysts }\end{array}$ & ES lines (\%) \\
\hline IVF blastocysts & 120 & $6(5)$ \\
IVF aggregated $(3 \times)$ blastocysts & 32 & $3(9.4)$ \\
In vivo produced blastocysts & 3 & $2(66.7)$ \\
\hline
\end{tabular}

IVF, in vitro fertilized.

that developed to the $1 \times, 2 \times$, and $3 \times$ blastocyst stages are shown in Table 3 . The aggregation rates of the $2 \times$ and $3 \times$ aggregates were not different $(78.4 \%$ for $2 \times$ vs $84.8 \%$ for $3 \times) 24 \mathrm{~h}$ after aggregation. However, development to the blastocyst stage increased significantly in the aggregated embryos $(82.8 \%$ for $2 \times$ and $84.5 \%$ for $3 \times)$ compared with that of the non-aggregated embryos $(46.8 \%$ for $1 \times)(p<$ $0.05)$. Moreover, the morphology of the $2 \times$ and $3 \times$ aggregated blastocysts was superior to that of nonaggregated blastocysts (Figure 2). When non-aggregated and aggregated embryos were seeded onto a feeder layer, the rate of blastocyst attachment was similar between the non-aggregated and aggregated blastocysts of each group ( $70 \%$ to $80 \%$; data not shown). As shown in Table 2, more cell lines were derived from the $3 \times$ aggregate group $(9.4 \%)$ than from the single embryo group (5\%).

\section{Verification of undifferentiated state and differentiation potential from outgrowths}

We investigated pluripotent marker expression in all cell lines. As shown Figure 3A, all cell lines expressed the Oct4, Sox2, and Nanog pluripotent marker genes, which play crucial roles as transcription factors in mESCs (Chambers et al., 2003; Masui et al., 2007). Our cell lines showed similar primary colony morphology and typical morphology and AP activity (Figure 3B). As shown in Figure 3C, we confirmed Oct4, Sox2, and Nanog expression in our cell lines by ICC analysis. We investigated EB formation and differentiation capacity into the three germ layers, such as endoderm, mesoderm, and ectoderm. As shown in Figure $4 \mathrm{~A}$ and $4 \mathrm{~B}$, the cell lines demonstrated normal karyotypes and the potential to form EBs when induced to differentiate after 5 days of culture using the hanging drop method. We

Table 3. Effect of embryo aggregation on the formation of in vitro-fertilized (IVF) blastocysts

\begin{tabular}{lccc}
\hline \multirow{2}{*}{ Embryo } & \multicolumn{3}{c}{ No. of embryos (\%) } \\
\cline { 2 - 4 } & Total & Aggregates & Blastocysts \\
\hline $1 \times$ IVF & 111 & n.a & $52(46.8)^{\mathrm{a}}$ \\
$2 \times$ IVF & 148 & $58 / 74(78.4)$ & $48 / 58(82.8)^{\mathrm{b}}$ \\
$3 \times$ IVF & 297 & $84 / 99(84.8)$ & $71 / 84(84.5)^{\mathrm{b}}$ \\
\hline
\end{tabular}

$1 \times$, zona-free, non-aggregated; $2 \times$, two-embryo aggregate; $3 \times$, threeembryo aggregate; n.a, not applicable.

Values with different letter superscripts within the same column are significantly different $(\mathrm{p}<0.05)$. 

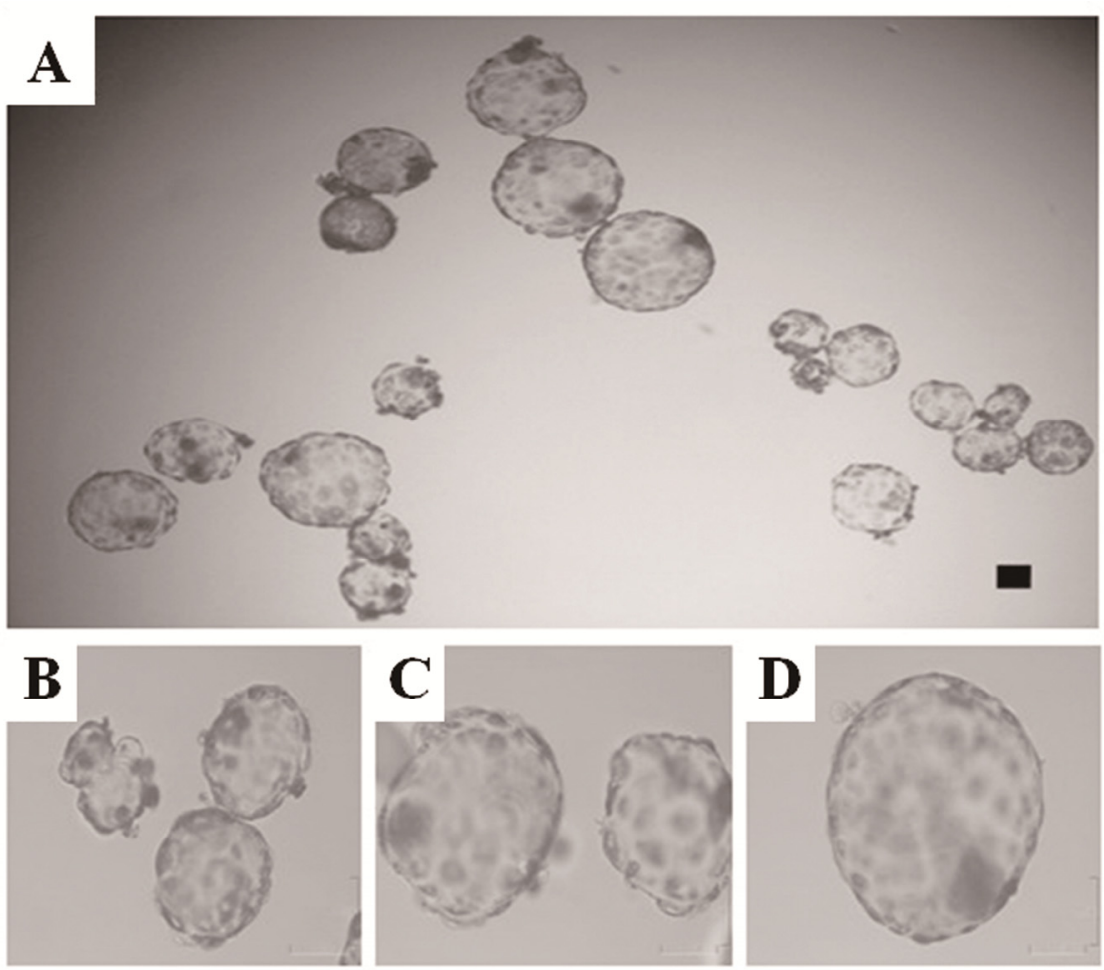

Figure 2. Morphology of $1 \times, 2 \times$, and $3 \times$ blastocysts. Morphology of $1 \times($ A: right; B), $2 \times($ A: left; C), and $3 \times($ A: above; D) blastocysts. In vitro-developed blastocysts from each group were collected on day 7 . This photograph was taken with a digital camera at the same scale. Scale bars $=100 \mu \mathrm{m}$. Abbreviations: $1 \times$, zona-free, non-aggregated; $2 \times$, two-embryo aggregate; $3 \times$, three-embryo aggregate

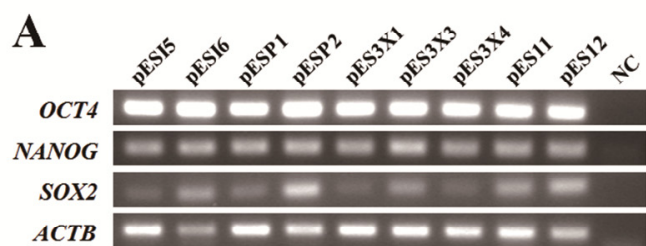

B

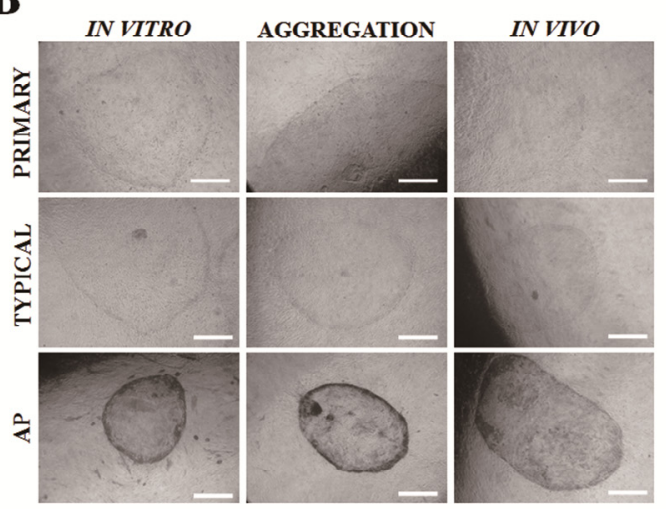

C
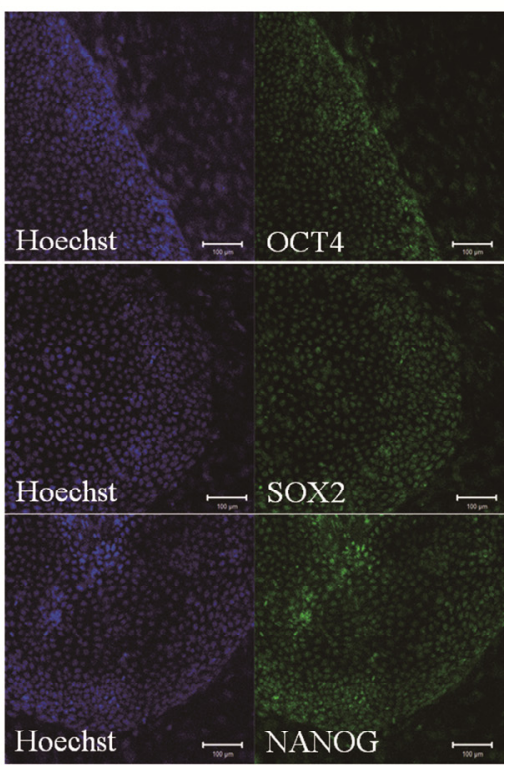

Figure 3. Verification of the undifferentiated state and differentiation potential of outgrowths. (A) Expression of pluripotent markers in our porcine embryonic stem (pES)-like cells. NC, water blank was used for negative control (NC). Several stem cell lines derived from various origins were used as a positive control. pESI5 and pESI6: derived from in vitro-fertilized embryos. pESP1 and pESP2: parthenogenetic embryos. pES11 and pES12: in vivo-produced embryos. (B) Primary colonies, typical morphology, and alkaline phosphatase (AP) activity in our pES-like cells, Scale bars $=200 \mu \mathrm{m}$. (C) Expression of the pluripotent markers Oct4, Sox2, and Nanog in pES-like cells by immunocytochemistry analysis. Scale bars $=100 \mu \mathrm{m}$. 


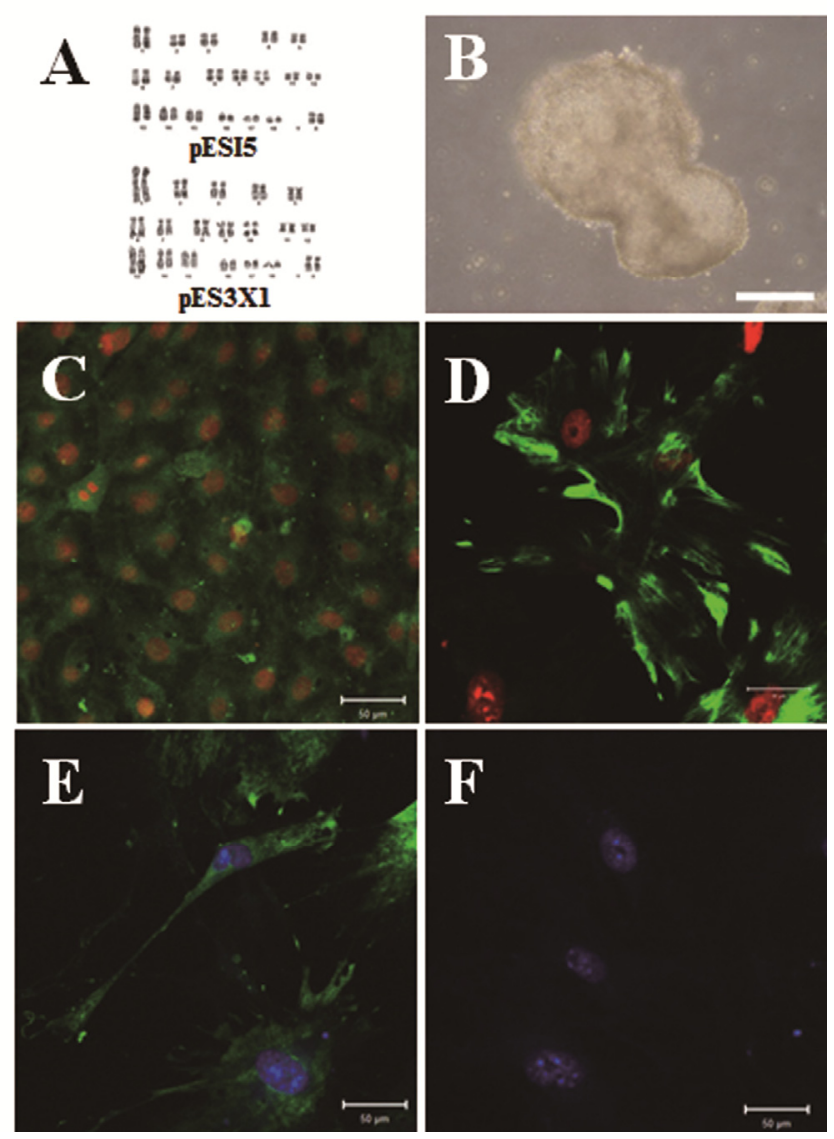

Figure 4. Karyotyping and differentiation potential of porcine embryonic stem (pES)-like cells. (A) Both the pES-like cells derived from in vitro fertilization (IVF) and IVF aggregated blastocysts had normal karyotypes. (B) Representative embryoid bodies (EBs) derived from pES-like cells after 5 days of culture using the hanging drop method. The differentiation potential of EBs cultured on culture plates into the three germ layers from pES-like cells was confirmed. (C) Cytokeratin17 (endoderm), (D) vimentin (mesoderm), and (E) neurofilament (ectoderm). (F) Nanog was the negative control. Scale bars $=100 \mu \mathrm{m}$.

observed a variety of differentiated cells after 1 to 2 weeks, when EBs were cultured on plates coated with $0.1 \%$ gelatin. As shown in Figure 4C to 4E, we confirmed the expression of three germ layer markers, including cytokeratin17 (endoderm), vimentin (mesoderm), and neurofilament (ectoderm) from differentiated cells in the ICC analysis. Nanog was not expressed in differentiated cells. These results indicate that the cell lines possess self-renewal and differentiation potential.

\section{DISCUSSION}

Blastocysts comprise two cell populations during embryogenesis (Friel et al., 2005). The first is the ICM, which subsequently develops into the epiblast and hypoblast; the epiblast forms the fetus. Therefore, the epiblast is referred to as a pluripotent cell population due to its capacity to generate all lineages, and it has been utilized as an ESC source. The other is the TE, which exists in an outer layer of blastocyst cells and gives rise to the fetal placenta. We observed the early process of generating ESCs from porcine embryos to understand the process of establishing ESC lines from porcine embryos. As shown in Figure $1 \mathrm{~A}$ and $1 \mathrm{~B}$, we observed two cell types in porcine blastocysts under high magnification; giant cells were found in the outer layer of the TE, and an ICM-like small cell mass existed on the interior of blastocysts. These two cell types were observed on the feeder cell layer 2 to 3 days after seeding the blastocysts (Figure 1C and 1D). However, the presumptive TE cells disappeared gradually, and ES-like primary cells were seen on the feeder cells about 7 days after seeding the embryos. As in our previous report (Park et al., 2013), we observed mouse ES-like colonies with a compact and dome-shape morphology at the early primary colony stage. However, the dome-shape morphology of the early primary colonies tended to differentiate into epithelial-like cells with a flat morphology after 2 to 3 days (Figure 1E and 1F). These flat ES-like cells were stably maintained for a long period without losing stemness or self-renewal potential. Further studies on optimal culture conditions to suppress differentiation into the primed state from the naïve pluripotent state are needed to establish authentic pESCs.

A remaining question is why it is more difficult to derive ESCs from other than in vivo-produced embryos. We hypothesized that the competence of the starting material affects poor derivation of pESCs. The embryo aggregation technique was applied to overcome the suboptimal pig embryo culture conditions. Aggregation of four-cell-stage porcine embryos efficiently supported in vitro blastocyst development, which resulted in a greater number of ES-like cell colonies formed than in non-aggregated blastocysts under the same culture conditions.

When aggregation occurs before the cell lineage forms, cells from each embryo can contribute to all cell lineages of the resulting embryos (Nagy and Rossant, 2001). Interactions between these cells are more prominent; thus, these cells are more likely to be involved in complementation (Boediono et al., 1999; Emerson et al., 2000). One way to achieve complementation is through cell-to-cell communication, such as gap or tight junctions, and another is through non-cell autonomous factors, such as trophic factors that stimulate signaling in neighboring cells (Zhou et al., 2008). Therefore, complementation via intercellular signaling between aggregated embryos can affect in vitro development. In our previous report (Lee et al., 2007), the four-cell stage was the best stage for aggregation in pigs. Moreover, aggregating IVF embryos enhances in vitro development at the pre-implantation stage. Similar results have been obtained after aggregating bovine 
cloned embryos at the four-cell stage (Zhou et al., 2008).

If aggregation of four-cell-stage embryos enhances in vitro development at the pre-implantation stage, it could influence isolation of ESCs derived from in vitro-produced blastocysts. Blastocyst quality is a very important factor when isolating ESCs for subsequent colony formation. Although porcine blastocysts have been obtained successfully using IVF and SCNT (Abeydeera and Day, 1997; Lee et al., 2003), their quality remains lower than that of in vivo-produced blastocysts. Obtaining a total cell number similar to that of in vivo-derived blastocysts is a valuable indicator of the viability of in vitro-produced embryos (Fuente and King, 1997). The total cell numbers of in vivo-derived blastocysts on days 6 and 7 were reported to be about 57 (Yoshioka et al., 2002), and 93 (de la Fuente and King, 1997), respectively, which increased to approximately 175 just before hatch (Davis, 1985). Our previous report (Lee et al., 2007) demonstrated that aggregation of four-cell-stage porcine embryos increased the number of cells with a normal ratio of ICM cells to total cells. The average mean cell numbers of $2 \times$ and $3 \times$ aggregated blastocysts were 129 and 169 , respectively. The $3 \times$ aggregated embryos appeared to have similar numbers to those of blastocysts derived in vivo just before hatch, and even exceeded the number of cells in in vivo-derived blastocysts on day 7. Deriving outgrowth lines from in vitro-produced embryos can be helpful. Actually, our results show that the derivation efficiency of the cell lines in the $3 \times$ aggregate group was two times higher than that of the single-embryo group (Table 2). Therefore, the embryoaggregation method effectively overcame the obstacles of an in vitro system to derive ESCs from IVF embryos. Interestingly, pESCs derived from $3 \times$ aggregated embryos at passage 16 had normal sex karyotypes. In our previous study (Lee et al., 2007), porcine IVF aggregates developed into chimeric blastocysts with random cell populations in each embryo. All embryos developed equally into chimeric pig blastocysts, and no particular embryo type was predominant.

In conclusion, we observed a change from a domeshaped morphology to a flat morphology during the early process of generating porcine embryo outgrowths. We demonstrated that aggregating four-cell-stage IVF embryos successfully supported in vitro development of porcine blastocysts during the pre-implantation stage and that these aggregated blastocysts formed more colonies than did their non-aggregated counterparts. We also showed that these cell lines had AP activity and expressed pluripotent markers such as Oct4, Sox2, and Nanog, The cell lines also formed EBs, revealed normal karyotypes, and were capable of differentiating into the three germ layers. This technique could be useful for therapeutic cloning, genetic engineering, and enhancing the quality of SCNT embryos.

\section{CONFLICT OF INTEREST}

We certify that there is no conflict of interest with any financial organization regarding the material discussed in the manuscript.

\section{ACKNOWLEDGMENTS}

This work was supported by the Next-generation BioGreen 21 Program (PJ01130012015), Rural Development Administration, Republic of Korea.

\section{REFERENCES}

Abeydeera, L. R. and B. N. Day. 1997. Fertilization and subsequent development in vitro of pig oocytes inseminated in a modified tris-buffered medium with frozen-thawed ejaculated spermatozoa. Biol. Reprod. 57:729-734.

Alberio, R., N. Croxall, and C. Allegrucci. 2010. Pig epiblast stem cells depend on activin/nodal signaling for pluripotency and self-renewal. Stem Cells Dev. 19:1627-1636.

Bavister, B. 2004. The role of animal studies in supporting human assisted reproductive technology. Reprod. Fertil. Dev. 16:719728.

Boediono, A., M. Ooe, M. Yamamoto, M. Takagi, S. Saha, and T. Suzuki. 1993. Production of chimeric calves by aggregation of in vitro fertilized bovine embryos without zonae pellucidae. Theriogenology 40:1221-1230.

Boediono, A., T. Suzuki, L. Y. Li, and R. A. Godke. 1999. Offspring born from chimeras reconstructed from pathenogenetic and in vitro fertilized embryos. Mol. Reprod. Dev. 53:159-170.

Boiani, M., S. Eckardt, N. A. Leu, H. R. Schöler, and K. J. McLaughlin. 2003. Pluripotency deficit in clone overcome clone-clone aggregation : epigenetic complementation? EMBO J. 22:5304-5312.

Chambers, I., D. Colby, M. Robertson, J. Nichols, S. Lee, S. Tweedie, and A. Smith. 2003. Functional expression cloning of Nanog, a pluripotency sustaining factor in embryonic stem cells. Cell 113:643-655.

Davis, D. L. 1985. Culture and storage of pig embryos. J. Reprod. Fertil. Suppl. 33:115-124.

de la Fuente, R. and W. A. King. 1997. Use of a chemically defined system for the direct comparison of inner cell mass and trophectoderm distribution in murine, porcine and bovine embryos. Zygote 5:309-320.

Emerson, M., A. R. Travis, R. Bathgate, T. Stojanov, D. I. Cook, E. Harding, D. P. Lu, and C. O'Neill. 2000. Characterization and functional significance of calcium transients in the 2-cell mouse embryo induced by an autocrine growth factor. The J. Biol. Chem. 275:21905-21913.

Friel, R., S. v. d. Sar and P. J. Mee. 2005. Embryonic stem cells: Understanding their history, cell biology and signalling. Adv. Drug Deliv. Rev. 57:1894-1903.

Keefer, C. L., D. Pant, L. Blomberg, and N. C. Talbot. 2007. Challenges and prospects for the establishment of embryonic stem cell lines of domesticated ungulates. Anim. Reprod. Sci. 98:147-168. 
Kim, H. S., G. S. Lee, S. H. Hyun, S. H. Lee, D. H. Nam, Y. W. Jeong, S. Kim, S. K. Kang, B. C. Lee, and W. S. Hwang. 2004. Improved in vitro development of porcine embryos with different energy substrates and serum. Theriogenology 61:1381-1393.

Kim, S., J. H. Kim, E. Lee, Y. W. Jeong, M. S. Hossein, S. M. Park, S. W. Park, J. Y. Lee, Y. I. Jeong, H. S. Kim, Y. W. Kim, S. H. Hyun and W. S. Hwang. 2010. Establishment and characterization of embryonic stem-like cells from porcine somatic cell nuclear transfer blastocysts. Zygote 18:93-101.

Lee, G. S., H. S. Kim, S. H. Hyun, D. Y. Kim, S. H. Lee, D. H. Nam, Y. W. Jeong, S. Kim, S. K. Kang, B. C. Lee, and W. S. Hwang. 2003. Improved developmental competence of cloned porcine embryos with different energy supplements and chemical activation. Mol. Reprod. Dev. 66:17-23.

Lee, S. G., C. H. Park, D. H. Choi, H. S. Kim, H. H. Ka, and C. K. Lee. 2007. In vitro development and cell allocation of porcine blastocysts derived by aggregation of in vitro fertilized embryos. Mol. Reprod. Dev. 74:1436-1445.

Li, M., Y. H. Li, Y. Hou, X. F. Sun, Q. Sun, and W. H. Wang. 2004. Isolation and culture of pluripotent cells from in vitro produced porcine embryos. Zygote 12:43-48.

Masui, S., Y. Nakatake, Y. Toyooka, D. Shimosato, R. Yagi, K. Takahashi, H. Okochi, A. Okuda, R. Matoba, A. A. Sharov, M. S. Ko, and H. Niwa. 2007. Pluripotency governed by Sox2 via regulation of Oct3/4 expression in mouse embryonic stem cells. Nat. Cell Biol. 9:625-635.

Nagy, A. and J. Rossant. 2001. Chimaeras and mosaics for dissecting complex mutant phenotypes. Int. J. Dev. Biol. 45:577-582.

Nagy, A., J. Rossant, R. Nagy, W. Abramow-Newerly, and J. C. Roder. 1993. Derivation of completely cell culture-derived mice from early-passage embryonic stem cells. Proc. Natl. Acad. Sci. USA. 90:8424-8428.
Neganova, I. E., G. G. Sekirina, and U. Eichenlaub-Ritter. 2000. Surface-expressed E-cadherin, and mitocondrial and microtuble distrbution in rescue of mouse embryos from 2cell block by aggregation. Mol. Hum. Reprod. 6:454-464.

Park, J. K., H. S. Kim, K. J. Uh, K. H. Choi, H. M. Kim, T. Lee, B. C. Yang, H. J. Kim, H. H. Ka, H. Kim, and C. K. Lee. 2013. Primed pluripotent cell lines derived from various embryonic origins and somatic cells in pig. PloS one 8:e52481.

Petters, R. M. and K. D. Wells. 1993. Culture of pig embryos. J. Reprod. Fertil. Suppl. 48:61-73.

Pickering, S. J., P. R. Braude, M. Patel, C. J. Burns, J. Trussler, V. Bolton, and S. Minger. 2003. Preimplantation genetic diagnosis as a novel source of embryos for stem cell research. Reprod. Biomed. Online 7:353-364.

Talbot, N. C. and A. Blomberg Le. 2008. The pursuit of ES cell lines of domesticated ungulates. Stem Cell Rev. 4:235-254.

Tang, P. C. and J. D. West. 2000. The effects of embryo stage and cell number on the composition of mouse aggregation chimaeras. Zygote 8:235-243.

Vassiliev, I., S. Vassilieva, L. F. Beebe, S. J. Harrison, S. M. McIlfatrick, and M. B. Nottle. 2010. In vitro and in vivo characterization of putative porcine embryonic stem cells. Cell. Reprogram. 12:223-230.

Wood, S. A., N. D. Allen, J. Rossant, A. Auerbach, and A. Nagy. 1993. Non-injection methods for the production of embryonic stem cell-embryo chimeras. Nature 365:87-89.

Yoshioka, K., C. Suzuki, A. Tanaka, I. M. Anas, and S. Iwamura. 2002. Birth of piglets derived from porcine zygotes cultured in a chemically defined medium. Biol. Reprod. 66:112-119.

Zhou, W., T. Xiang, S. Walker, R. V. Abruzzese, E. Hwang, V. Farrar, B. Findeisen, S. Sadeghieh, F. Arenivas, S. H. Chen, and I. Polejaeva. 2008. Aggregation of bovine cloned embryos at the four-cell stage stimulated gene expression and in vitro embryo development. Mol. Reprod. Dev. 75:1281-1289. 